\title{
Clinical Reasoning: A 68-Year-Old Man With Proximal Weakness and Seizures
}

Tychicus Chen, MD, FRCPC

Neurology ${ }^{\circledR}$ 2021;97:e423-e428. doi:10.1212/WNL.0000000000012137
Correspondence

Dr. Chen

tychicus@mail.ubc.ca

\section{Section 1}

A 68-year-old man developed progressive weakness over 4 weeks. He had difficulty rising from a low position, climbing stairs, and raising his arms overhead, particularly toward evenings. He had no pain, sensory, or bowel or bladder symptoms, and denied ptosis, diplopia, dysphagia, or dyspnea. He had mild unintentional weight loss but no fevers. His medical history revealed eczema and active smoking (50 pack-years). There was no family history of neuromuscular disorders.

On examination, vital signs were normal; the patient was alert, oriented, attentive, and fluent with no memory disturbance. Cranial nerves revealed symmetric, reactive pupils, full extraocular movements, facial strength, and no fatigable ptosis. Muscle bulk and tone were normal without fasciculations. Neck strength was full, with variable moderate weakness in shoulder and hip girdles, slightly worse with repetition. Deep tendon reflexes were absent except biceps and patella, which were reduced but present. Sensation and coordination were unremarkable.

\section{Questions for Consideration:}

1. What is the differential diagnosis for progressive weakness without sensory loss?

2. What clinical tests and investigations would you consider?

\section{GO TO SECTION 2}




\section{Section 2}

The patient presents with subacute painless, proximal, symmetric weakness over 4 weeks, with possible fatigability, importantly without craniobulbar or sensory abnormalities, and reflexes are reduced or absent. Main considerations include muscle or neuromuscular junction disorders. Motor neuropathy including acute motor axonal neuropathy and multifocal motor neuropathy with conduction block, as well as motor neuron disease such as primary muscular atrophy, are unlikely without reduced tone or fasciculations. Amyotrophic lateral sclerosis is unlikely without bulbar or upper motor neuron signs. Lack of sensory symptoms makes polyradiculopathy and peripheral neuropathy unlikely, as well as spinal cord disease without sphincter dysfunction or upper motor neuron signs; notable exceptions include acute flaccid myelitis from poliovirus, nonpolio enteroviruses, and West Nile virus. This patient had no recent travel or infectious prodrome. There were no corticobulbar signs or encephalopathy to suggest other central processes.

Muscle disorders may be inflammatory or noninflammatory, congenital or acquired. Inflammatory and toxic myopathies may present with myalgias; this patient had no pain or exposures. His age and subacute presentation are atypical for most muscular dystrophies or genetic myopathies, with no relevant family history, although some genetic conditions demonstrate low penetrance or anticipation, including certain limb-girdle muscular dystrophies or Pompe disease. Myopathies may include systemic involvement, such as cardiac dysfunction, rashes (dermatomyositis), myoclonus (mitochondrial myopathies), or myoglobinuria from muscle damage, which were not present. Acquired neuromuscular junction conditions can be divided into postsynaptic disorders like myasthenia gravis (MG), associated with thymoma or presynaptic disorders such as botulism - although unlikely without pupillary involvement-and Lambert-Eaton myasthenic syndrome (LEMS), associated with smoking and small cell lung cancer (SCLC), although much less common than MG. Whereas both MG and LEMS may present with proximal weakness, the clinical phenotype is more typical for LEMS. Ocular involvement, diurnal variation, and fatigability are characteristic of MG, whereas patients with LEMS may have improvement with repetition or the day's progression, as well as autonomic symptoms. Electrodiagnostics and serum creatine kinase levels can help distinguish the above.

On further history, the patient denied erectile dysfunction but complained of increasingly dry mouth. Repeat examination revealed postexercise facilitation of deep tendon reflexes. Serum biochemistry and creatine kinase were normal. Spinal imaging demonstrated no abnormalities. Nerve conduction studies confirmed normal sensory potentials and low compound muscle action potentials without motor conduction block, which improved with exercise (figure 1, A and B). Slow repetitive nerve stimulation (RNS) showed decrement (figure 1B), while fast RNS showed increment (figure 1C). Chest radiograph and CT showed minimal mediastinal and hilar lymphadenopathy with no suspicious pulmonary masses or thymoma. Serum voltage-gated calcium channel (VGCC) antibodies returned highly positive $(387.1 \mathrm{pmol} / \mathrm{L})$.

\section{Questions for Consideration:}

1. What is the etiology of LEMS?

2. What is the treatment for LEMS? 
A. Left ulnar

Wrist-ADM $5 \mathrm{mV} / \mathrm{D} 3 \mathrm{~ms} / \mathrm{D}$

Bl. elbow-ADM $5 \mathrm{mV} / \mathrm{D} 3 \mathrm{~ms} / \mathrm{D}$

Ab. elbow-ADM $5 \mathrm{mV} / \mathrm{D} 3 \mathrm{~ms} / \mathrm{D}$

B. Left ADQM


C. Left ADQM

Pre-activation below elbow, and above elbow: low distal amplitude $(4.6 \mathrm{mV})$, normal distal latency $(3.2 \mathrm{~ms})$, and mild slowing across the elbow without conduction block (53-39 m/s). (B) Slow repetitive nerve stimulation (RNS) (3 Hz, 10 stimulations): (a) before exercise, decremental response in amplitude (2.7-1.3 mV; $51 \%$ decrement) from stimulus 1 to 4 (blue bars); (b) following 60 seconds of maximum voluntary contraction, repair of motor amplitude (now $6.4 \mathrm{mV}$ ) demonstrating postexercise facilitation, but still decremental response in amplitude (6.4-5.1 mV; 21\% decrement) from stimulus 1 to 4 (blue bars). (C) Fast RNS (30 Hz, 90 stimulations): incremental response in amplitude (3.0-8.2 mV; $172 \%$ increment) between stimulus 20 and 90 (blue lines). $A D M=$ Abductor digiti minimi; $A D Q M=$ Abductor digiti quinti minimi. 


\section{Section 3}

LEMS is a presynaptic neuromuscular junction disorder characterized by inhibition of P/Q-type VGCCs that mediate calcium influx, which normally respond to membrane depolarization, leading to proximal weakness and autonomic dysfunction. ${ }^{1,2}$ Antibodies against VGCC are present in $85 \%-95 \%$ of cases, ${ }^{3}$ higher in paraneoplastic LEMS, which is highly associated with SCLC, found in $60 \%$ of cases, with smoking a strong risk factor. ${ }^{2}$ SCLC must be considered when LEMS is diagnosed, found on initial screening in most patients. However, tumors may begin small with undetectable parenchymal lung mass and only lymphadenopathy as in this case. Immunotherapy with corticosteroids, IV immunoglobulin (IVIg), and plasma exchange (PLEX) are used; however, as with all paraneoplastic syndromes, management relies on treatment of the underlying malignancy. ${ }^{1}$

Similar to how maximum voluntary contraction transiently increases intracellular calcium concentration, leading to postexercise facilitation and increment, symptomatic treatment targets augmentation of synaptic calcium, which increases release of acetylcholine quanta. ${ }^{1,4} 3,4$ Diaminopyridine (DAP) blocks presynaptic potassium channels, which prolongs depolarization, leading to increased calcium influx and intracellular calcium accumulation, which improve the ability of vesicles to fuse and release acetylcholine. ${ }^{4}$ Acetylcholinesterase inhibitors like pyridostigmine have modest benefit and are no longer first line. Guanidine increases intracellular calcium but is not widely available due to bone marrow suppression and nephrotoxicity. ${ }^{4}$

The patient was treated with IVIg and 3,4-DAP with improvement in strength. Although no pulmonary mass was seen, given strong suspicion, endobronchial ultrasound was pursued, and lymph node biopsy confirmed SCLC. Staging PET and MRI brain showed limited disease confined to the lungs without brain involvement. The patient started chemotherapy with carboplatin and etoposide with planned radiotherapy. He developed neutropenia, delaying chemotherapy, followed by confusion and seizures, and was brought to the hospital and intubated for status epilepticus.

\section{Questions for Consideration:}

1. What are potential causes of seizures in this patient?

2. What is your next step in management?

\section{GO TO SECTION 4}




\section{Section 4}

Infectious meningoencephalitis must be considered with neutropenia and chemotherapy. Seizures are a rare side effect of 3,4DAP from potentiation of synaptic action potentials, although reported with higher doses as CNS penetration is less than with 4-aminopyridine. ${ }^{4} \mathrm{New}$ seizures in a patient with malignancy should prompt consideration for brain metastases. Limbic encephalitis would also be a strong consideration with SCLC.

EEG demonstrated frequent periodic independent epileptiform discharges in the temporal regions bilaterally (figure $2 \mathrm{~A}$ ), with recurrent electrographic seizures, which continued despite prompt discontinuation of 3,4-DAP and addition of escalating antiepileptic drugs. Broad antimicrobial coverage was started, but CSF showed normal protein, no cells, negative viral studies, and was sent for paraneoplastic antibodies. Contrast MRI showed no abnormalities to suggest metastases or encephalitis (figure $2 \mathrm{~B}$ ).

PLEX was initiated for presumed limbic encephalitis, but seizures remained refractory without anesthetic infusions despite multiple antiepileptics. Although limbic encephalitis associated with SCLC should not be a reason to withdraw treatment, the family ultimately wished to withdraw care in respect of previous advance care directives following extensive counseling. A few days after the patient died, cell-based assay for $\gamma$-aminobutyric acid-B receptor $\left(\mathrm{GABA}_{\mathrm{B}} \mathrm{R}\right)$ antibodies returned highly positive from CSF.

\section{Question for Consideration:}

1. What is the significance of this result?

Figure 2 EEG and Brain Imaging
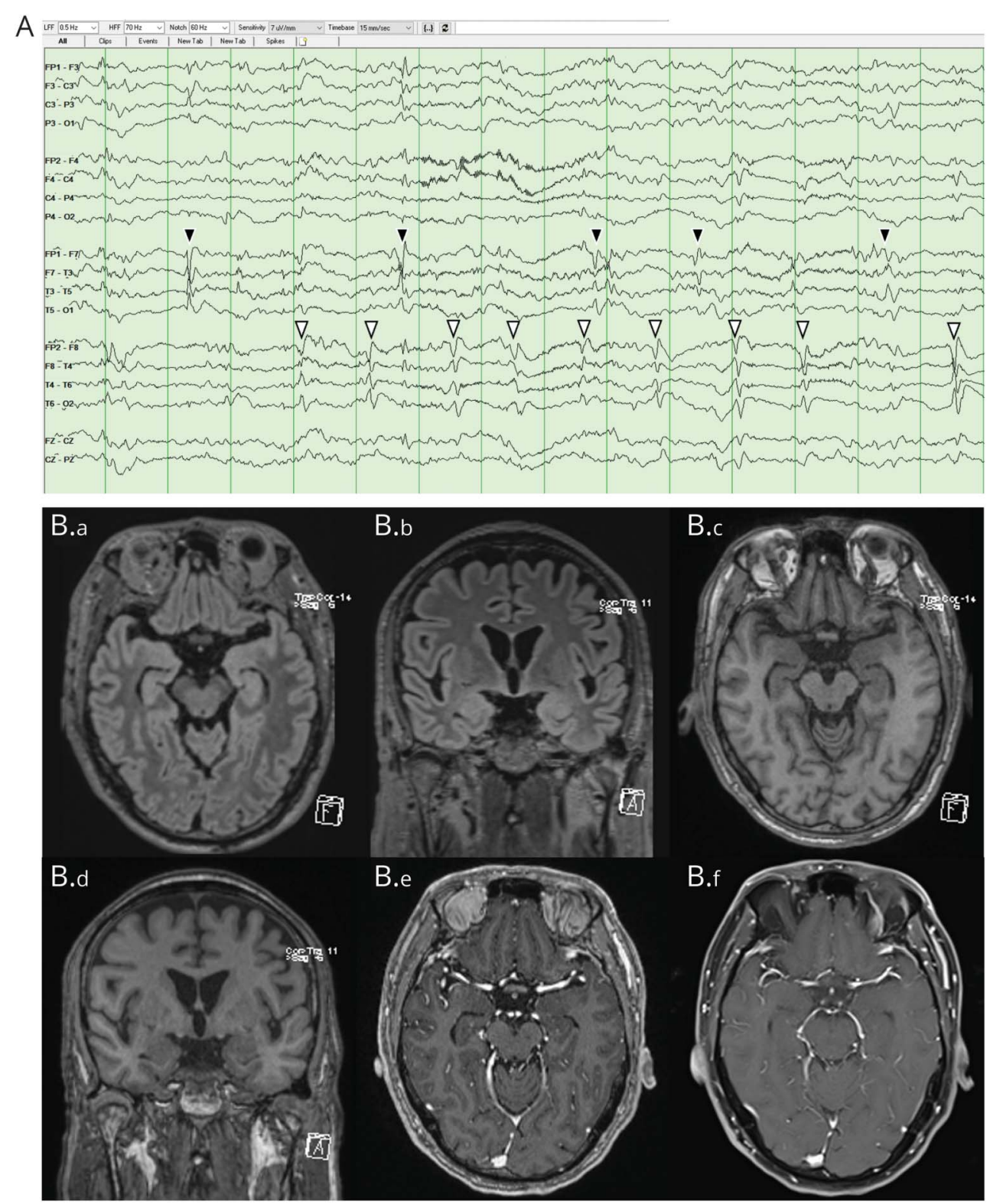
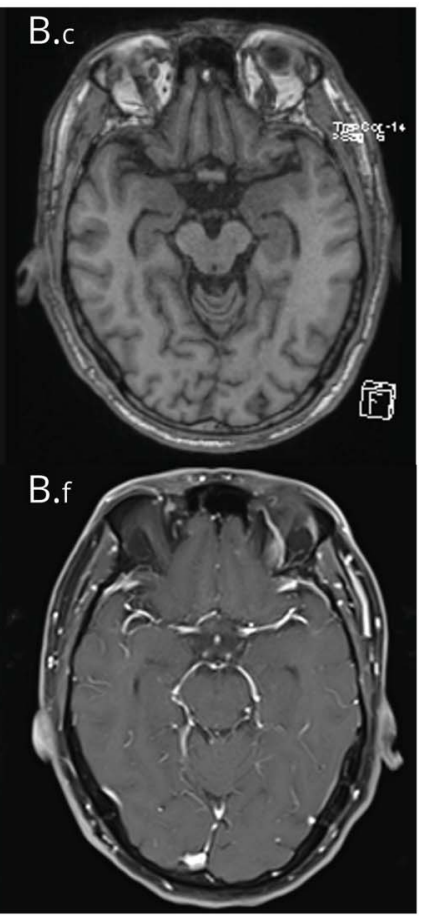

(A) EEG recorded in longitudinal bipolar montage shows frequent independent left temporal (black arrowheads) and right temporal (white arrowheads) epileptiform abnormalities. Time base $15 \mathrm{~mm} / \mathrm{s}$, sensitivity $7 \mu \mathrm{V} / \mathrm{mm}$, LF filter $0.5 \mathrm{~Hz}$, HF filter $70 \mathrm{~Hz}$. (B) MRI brain, T2fluid-attenuated inversion recovery (B.a) axial and (B.b) coronal views, and T1-weighted (B.C) axial and (B.d) coronal views, showing no temporal lobe abnormalities; axial (B.e) T1weighted post-gadolinium with (B.f) fat-saturated views, showing no abnormal enhancement. 


\section{Discussion}

This case highlights several important points. LEMS was clinically suspected early based on history and physical examination, highlighting the importance of clinical reasoning and signs such as postexercise facilitation. This was confirmed, which led to an aggressive search for underlying SCLC, leading to earlier tissue diagnosis and treatment, despite initial chest imaging interpreted as insignificant. The most important prognostic factor for SCLC is cancer stage at diagnosis, with median survival ranging 4-11 months. ${ }^{5}$ Some studies have shown longer survival in SCLC with paraneoplastic LEMS, likely from earlier diagnosis and treatment or from other immune-mediated effects. ${ }^{4,5}$

Clinicians should be aware of side effects for medications prescribed, especially less commonly used medications like 3,4-DAP. In this case, although it was prudent to stop the drug immediately when the patient presented with seizures, cognitive bias should be avoided in assuming this as the sole cause. It is always important to consider a broad differential diagnosis, especially when patients with a typical presentation develop atypical symptoms, as the diagnosis of anti-GABA $A_{B} R$ encephalitis was unexpected. $G_{A B A} R$ are guanine nucleotide-binding protein-coupled metabotropic receptors for the inhibitory neurotransmitter GABA and are expressed broadly throughout the nervous system. ${ }^{6}$ Anti$\mathrm{GABA}_{\mathrm{B}} \mathrm{R}$ antibodies have been implicated in paraneoplastic limbic encephalitis, although only around 100 cases have been reported since 2010, and are also associated with SCLC in up to $50 \%{ }^{7,8}$ In the largest cohort to date of 32 patients, seizures occurred in $90 \%$, while $42 \%$ developed superrefractory status epilepticus. ${ }^{7}$ MRI showed mesial temporal changes in only $44 \%$, whereas EEG was abnormal in $84 \%$ with epileptic and encephalopathic findings in $44 \%{ }^{7}$ One patient also had LEMS, with proximal weakness preceding encephalitis by 9 months. ${ }^{7}$

The co-occurrence of LEMS and anti-GABA $\mathrm{B}_{\mathrm{B}} \mathrm{R}$ encephalitis in the setting of SCLC is rare. It has also been described in a case report where seizures occurred before initiation of 3,4-DAP and the authors suggest broad screening for paraneoplastic antibodies when treating LEMS. ${ }^{9}$ However, anti-GABA $A_{B} R$ antibodies have been demonstrated in patients with SCLC without encephalitis, ${ }^{10}$ and one study found no anti-GABA $A_{B} R$ antibodies in 116 patients with SCLC without neurologic symptoms, although this was in serum. ${ }^{8}$ Coexistent paraneoplastic syndromes with concurrent autoantibodies are exceptionally rare, but it is important to remain diligent for multiple paraneoplastic syndromes in a single patient.

The prognostic significance of anti-GABA $A_{B} R$ encephalitis coinciding with paraneoplastic LEMS is unknown given the rarity, but the few cases reported seem to show stabilization of symptoms with immunotherapy and cancer treatment. ${ }^{7,9}$ Prolonged intubation can occur as status epilepticus and neuromuscular respiratory failure are both reasons for ventilatory support. One wonders if more aggressive immunotherapy may have been successful in this case, but seizures remained refractory to multiple antiepileptics and PLEX, and the patient's and family's wishes had to be respected. However, paraneoplastic limbic encephalitis associated with SCLC can respond to treatment and should not be a reason to withhold oncotherapy. In the cohort of 32 patients mentioned above, the majority had cognitive improvement and seizure freedom; the median survival was 17 months. ${ }^{7}$ In another series of 28 patients with anti-GABA $A_{B} R$ encephalitis without LEMS, mortality was $32 \%$ and median survival 6.5 months; tumor progression was the most common cause of death and status epilepticus in $1 .{ }^{11}$ Onset above age 45 and presence of tumor were associated with higher risk. ${ }^{11}$

This case demonstrates challenging decisions clinicians may face without complete information when certain results are not immediately available or access to specialized testing is limited.

\section{Acknowledgment}

The author thanks the patient and his family. The author also thanks Dr. Jennifer Percy, Division of Neurology, Department of Medicine, University of British Columbia, for provision of EEG images.

\section{Study Funding}

The author reports no targeted funding.

\section{Disclosure}

T. Chen reports no disclosures. Go to Neurology.org/N for full disclosures.

\section{References}

1. Titulaer MJ, Lang B, Verschuuren JJ. Lambert-Eaton myasthenic syndrome: from clinical characteristics to therapeutic strategies. Lancet Neurol. 2011;10(12): 1098-1107.

2. O'Neill JH, Murray NM, Newsom-Davis J. The Lambert-Eaton myasthenic syndrome: a review of 50 cases. Brain. 1988;111(pt 3):577-596.

3. Lennon VA, Kryzer TJ, Griesmann GE, et al. Calcium-channel antibodies in the Lambert-Eaton syndrome and other paraneoplastic syndromes. N Engl J Med. 1995; 332(22):1467-1474

4. Lindquist S, Stangel M. Update on treatment options for Lambert-Eaton myasthenic syndrome: focus on use of amifampridine. Neuropsychiatr Dis Treat. 2011;7: 341-349.

5. Maddison P, Gozzard P, Grainge MJ, Lang B. Long-term survival in paraneoplastic Lambert-Eaton myasthenic syndrome. Neurology. 2017;88(14) 1334-1339.

6. Benarroch EE. GABAB receptors: structure, functions, and clinical implications. Neurology. 2012;78(8):578-584.

7. van Coevorden-Hameete MH, de Bruijn MAAM, de Graaff E, et al. The expanded clinical spectrum of anti-GABABR encephalitis and added value of KCTD16 autoantibodies. Brain. 2019;142(6):1631-1643.

8. Höftberger R, Titulaer MJ, Sabater L, et al. Encephalitis and GABAB receptor antibodies: novel findings in a new case series of 20 patients. Neurology. 2013;81(17): 1500-1506.

9. Cho JJ, Wymer JP. Paraneoplastic Lambert-Eaton myasthenic syndrome with limbic encephalitis: clinical correlation with the coexistence of anti-VGCC and anti-GABAB receptor antibodies. J Clin Neuromuscul Dis. 2017;19(2):84-88.

10. Dogan Onugoren M, Rauschka H, Bien CG. Conjoint occurrence of GABAB receptor antibodies in Lambert-Eaton myasthenic syndrome with antibodies to the voltage gated calcium channel. J Neuroimmunol. 2014;273(1-2):115-116.

11. Lin J, Li C, Li A, et al. Encephalitis with antibodies against the GABAB receptor: high mortality and risk factors. Front Neurol. 2019;10:1030. 


\section{Neurology}

\section{Clinical Reasoning: A 68-Year-Old Man With Proximal Weakness and Seizures}

Tychicus Chen

Neurology 2021;97;e423-e428 Published Online before print April 30, 2021

DOI 10.1212/WNL.0000000000012137

This information is current as of April 30, 2021

\section{Updated Information \&} Services

References

Subspecialty Collections

\section{Permissions \& Licensing}

Reprints including high resolution figures, can be found at: http://n.neurology.org/content/97/4/e423.full

This article cites 11 articles, 3 of which you can access for free at: http://n.neurology.org/content/97/4/e423.full\#ref-list-1

This article, along with others on similar topics, appears in the following collection(s):

\section{Clinical neurology examination}

http://n.neurology.org/cgi/collection/clinical_neurology_examination EMG

http://n.neurology.org/cgi/collection/emg

Lambert-Eaton syndrome

http://n.neurology.org/cgi/collection/lamberteaton_syndrome

Paraneoplastic syndrome

http://n.neurology.org/cgi/collection/paraneoplastic_syndrome

Status epilepticus

http://n.neurology.org/cgi/collection/status_epilepticus

Information about reproducing this article in parts (figures,tables) or in its entirety can be found online at:

http://www.neurology.org/about/about_the_journal\#permissions

Information about ordering reprints can be found online:

http://n.neurology.org/subscribers/advertise

Neurology ${ }^{\circledR}$ is the official journal of the American Academy of Neurology. Published continuously since 1951, it is now a weekly with 48 issues per year. Copyright (C) 2021 American Academy of Neurology. All rights reserved. Print ISSN: 0028-3878. Online ISSN: 1526-632X.



